\title{
Avaliação da substituição de placas de PMMA por PLA impresso 3D na resposta TL do CaSO4:Dy
}

\author{
S. B. de Almeida ${ }^{\text {a }}$ C. C. S, Valeriano ; L. L. Campos; \\ a, Gerência de Metrologia das Radiações. Instituto de Pesquisas Energéticas e Nucleares - \\ IPEN/CNEN, 05508-000, São Paulo-SÃO PAULO, Brasil \\ caio144fisicamedica@gmail.com
}

\begin{abstract}
RESUMO
$\mathrm{O} \mathrm{CaSO}_{4}$ :Dy é um material dosimétrico produzido nacionalmente, que apresenta alta sensibilidade, porém, uma alta dependência energética da resposta termoluminescente (TL) na região do efeito fotoelétrico. O objetivo deste trabalho é realizar um estudo comparativo da resposta TL do CaSO4:Dy quando irradiado com diferentes energias, na presença de placas de ácido polilático (PLA) impressas 3D e polimetilmetacrilato (PMMA). Os resultados obtidos indicam que o PLA apresenta uma diferença na atenuação da radiação, comparada ao PMMA, indicando que deve ser levada em consideração a qualidade da impressão empregada na confecção da placa a ser irradiada.
\end{abstract}

Palavras-chave: Impressora 3D, Dosímetro termoluminescente, Dependência energética.

\begin{abstract}
The CaSO4:Dy is a dosimetric material that is nationally produced, which presents high sensibility but a high energy dependence of its thermoluminescent (TL) response at the photoelectric effect region. This study aims to compare the TL response of the CaSO4:Dy when irradiated with different energies at the presence of 3D printed plates of polylactic acid (PLA) and polymethilmetacrylate (PMMA). Results show that the PLA presents different radiation attenuation when compared to PMMA, indicating that the quality of the print used on PLA plates manufacture should be taken into account.
\end{abstract}

Keywords: 3D printer, thermoluminescent dosimeter, energetic dependence. 


\section{INTRODUÇÃO}

Sempre que um fósforo termoluminescente (TL) é irradiado com feixe de fótons, seja para calibração ou para medição, deve ser levada em consideração a condição de equilíbrio eletrônico, normalmente expressa em massa por área $\left(\mathrm{mg} / \mathrm{cm}^{2}\right)$. Materiais como PMMA, Teflon e Nylon são normalmente utilizados para obtenção de equilíbrio eletrônico, sendo recomendado colocar uma espessura maior de material do que a requerida, de modo a reduzir o erro produzido na irradiação.

O CaSO4:Dy (figura 1) é um material desenvolvido, patenteado e produzido pelo IPEN, comercializado em forma de pó e pastilhas de $6 \mathrm{~mm}$ de diâmetro e espessura de 0,2 e 0,8 $\mathrm{mm}$, com massa respectivamente de 10 e $50 \mathrm{mg}$. Embora apresente alta sensibilidade, devido ao seu número atômico efetivo alto $(15,3)$, apresenta também uma alta dependência energética da resposta TL na região de efeito fotoelétrico.

Figura 1. Pastilhas dosimétricas de sulfato de cálcio dopado com disprósio (CaSO $4: D y)$.

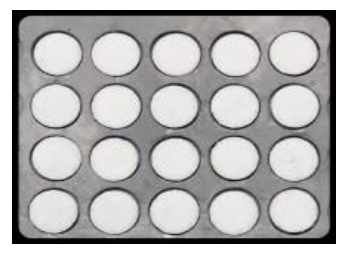

As principais propriedades dosimétricas do sulfato de cálcio são apresentadas na tabela 1 .

Tabela 1: Propriedades dosimétricas do $\mathrm{CaSO}_{4}: \mathrm{Dy}$.

\begin{tabular}{lc}
\hline Material ativador & Dy \\
Número atômico efetivo $(\mathbf{Z})$ & 15,3 \\
Densidade $\left[\right.$ g.cm $^{-3}$ ] & 4,93 \\
\hline
\end{tabular}




\begin{tabular}{ll}
\hline Decaimento térmico do pico dosimétrico à $\mathbf{2 0}^{\circ} \mathbf{C}$ & $5 \%$ ano \\
Tratamento pré-irradiação & $300^{\circ} \mathrm{C} / 3 \mathrm{~h}$
\end{tabular}

Visando suprir as novas necessidades do mercado, a partir dos anos 80, teve início a utilização das técnicas de prototipagem rápida. O emprego dessa tecnologia, em seus primórdios, era com a finalidade de confecção rápida de protótipos, porém, se expandiu para áreas como saúde e produção. Atualmente, a essa tecnologia é dado o nome de fabricação por adição (AM Additive Manufacturing).

Também chamada de impressão 3D, a fabricação por adição caracteriza-se por ser o processo no qual, objetos físicos de três dimensões (3D) são confeccionados através da transformação de objetos virtuais em objetos sólidos por meio de uma impressora 3D.

Uma impressora 3D, basicamente, interpreta um desenho 3D elaborado em qualquer sistema CAD que exporte em formato STL e o transforma em um objeto físico por meio da construção camada por camada, até que esteja finalizada sua produção. Dentre os materiais usados em impressora 3D podem ser citados o PLA (ácido polilático), cuja densidade varia de $1210 \mathrm{~kg} / \mathrm{m}^{3}$ a $1430 \mathrm{~kg} / \mathrm{m}^{3}$.

No presente trabalho foi avaliada a resposta termoluminescente do $\mathrm{CaSO}_{4}$ :Dy, empregando placas de PLA impressas em 3D e de polimetilmetacrilato (PMMA) de mesma espessura para obtenção da condição de equilíbrio eletrônico.

\section{MATERIAIS E MÉTODOS}

No presente trabalho foram empregadas:

- $\quad$ Placas de polimetilmetacrilato (PMMA) com $3 \mathrm{~mm}$ de espessura;

- Placas de ácido polilático (PLA), impressas por uma impressora 3D, com 3 mm de espessura (figura 2b). 
- 30 pastilhas dosimétricas de CaSO4:Dy previamente selecionadas, apresentando sensibilidade dentro de $\pm 5 \%$.

Também foram empregados:

- $\quad$ Impressora 3D UP Modelo Plus 2 (figura 2a);

Figura 2. Impressora 3D UP Modelo Plus 2 (a); e placa de PLA impressa (b).
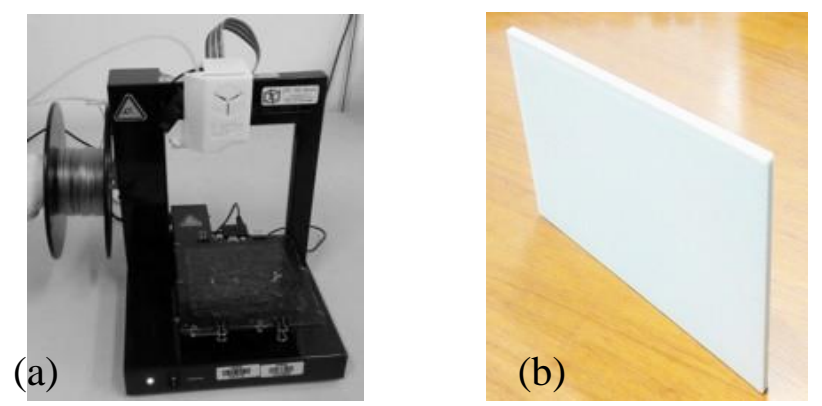

- $\quad$ Leitora termoluminescente Thermo Scientific Harshaw, modelo 4500;

- Irradiador Panorâmico de ${ }^{137} \mathrm{Cs}$ marca Hopewell, com atividade de 38,11 GBq em 17/11/2014 (Figura 3);

Figura 3. Irradiador Panorâmico de ${ }^{137} \mathrm{Cs}$ Hopewell.

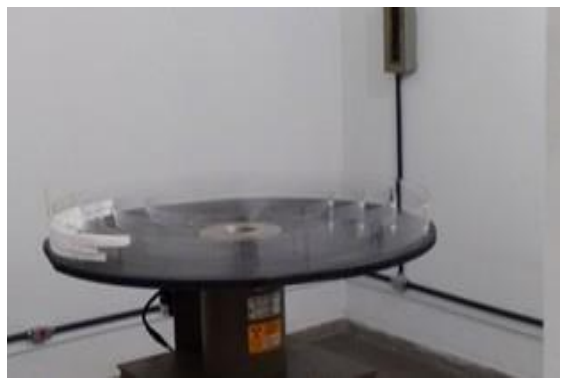

- $\quad$ Fonte de radiação gama do ${ }^{60} \mathrm{Co}$, com atividade de $0,339 \mathrm{TBq}$ em setembro de 1999 ;

- Sistema de Raios X Pantak/Seifert com as qualidades de radioproteção e radiodiagnóstico apresentadas na Tabela 2. 
Tabela 2: Qualidades de Raios X do Sistema de Raios X Pantak/Seifert

\begin{tabular}{c|c|c|c}
\hline $\begin{array}{c}\text { Qualidade } \\
\text { da Radiação }\end{array}$ & $\begin{array}{c}\text { Tensão } \\
(\mathbf{k V})\end{array}$ & CSR & $\begin{array}{c}\text { Energia } \\
\text { Efetiva (keV) }\end{array}$ \\
\hline RQR3 & 50 & $1,78 \mathrm{mmAl}$ & 29,7 \\
RQR5 & 70 & $2,58 \mathrm{mmAl}$ & 34 \\
RQR10 & 150 & $6,57 \mathrm{mmAl}$ & 46,5 \\
N150* & 150 & $2,4 \mathrm{mmCu}$ & 118 \\
\hline
\end{tabular}

*qualidade de radioproteção

As placas de PLA empregadas neste trabalho foram desenhadas utilizando o software FreeCAD e impressas no modo de menor preenchimento interno. A representação do preenchimento pode ser visto na figura 4. As energias de raios X utilizadas são empregadas na calibração de equipamentos de diagnóstico e radioproteção. Para todas as energias, exceto para a qualidade $\mathrm{N} 150$, o tempo de exposição foi calculado para uma dose de $30 \mathrm{mGy}$. Esse valor de dose foi escolhido por fazer parte da curva de calibração dos dosímetros utilizados. Em razão das limitações da taxa de dose da qualidade N150, a dose aplicada foi de $10 \mathrm{mGy}$. As doses de radiação gama do ${ }^{137} \mathrm{Cs} \mathrm{e}{ }^{60} \mathrm{Co}$ foram também de $30 \mathrm{mGy}$.

Figura 4. Modo de preenchimento da placa de PLA impressa

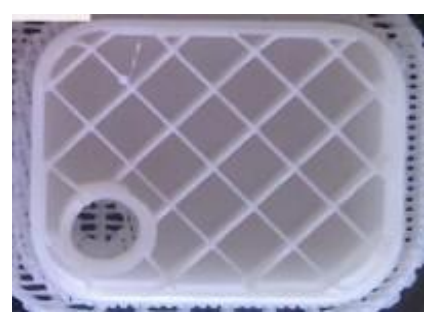




\section{RESULTADOS E DISCUSSÕES}

Os dosímetros TL de $\mathrm{CaSO}_{4}$ :Dy utilizados foram previamente selecionados (em lote) com resposta TL dentro de $+0,2 \%$ (média ponderada pela incerteza), sendo sua curva de resposta TL em função da dose para radiação gama do ${ }^{60} \mathrm{Co}$, mostrada na figura 5 , que demonstra a linearidade da resposta TL no intervalo de dose estudado.

Figura 5. Curva de resposta TL do CaSO4:Dy em função da dose para radiação gama do ${ }^{60} \mathrm{Co}$.

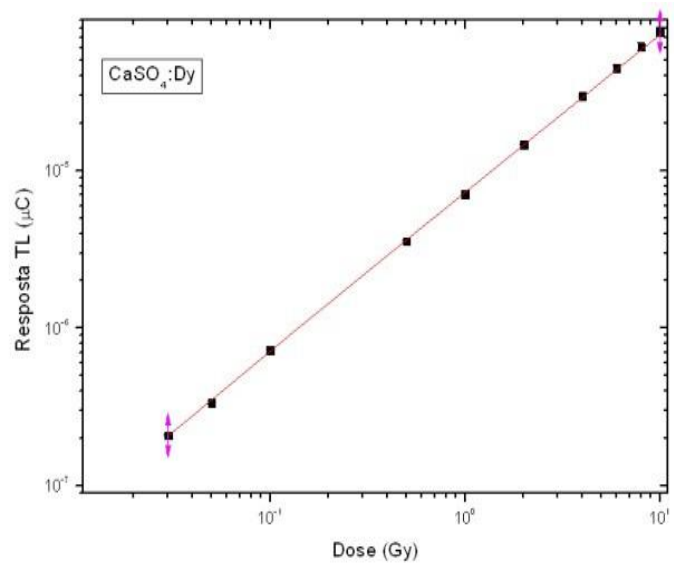

Os resultados obtidos com as placas de PLA de $3 \mathrm{~mm}$ de espessura foram comparados com os resultados obtidos com as placas de $3 \mathrm{~mm}$ de espessura de PMMA (material utilizado como espessura de equilíbrio eletrônico na seleção e calibração do $\mathrm{CaSO}_{4}$ :Dy com radiação gama do $\left.{ }^{60} \mathrm{Co}\right)$.

As respostas TL obtidas para as diferentes energias foram relacionadas com a resposta TL obtida para o ${ }^{60} \mathrm{Co}$, de modo a gerar os fatores de correção para dependência energética da resposta, por unidade de dose. As tabelas $3 \mathrm{a}$ e $3 \mathrm{~b}$ apresentam, respectivamente, os valores de leitura e fatores de correção para dependência energética obtidos para o PMMA e para o PLA. 
Os fatores de correção apresentados nas tabelas 3a e 3b foram obtidos por meio da razão da resposta TL pela dose (RTL/dose) para cada energia. A partir desses valores, foi também calculado, para cada energia, o fator de correção por meio da equação 1 .

$$
F_{\text {Correĝ̣a }}=\frac{R_{T L}}{\text { dose }} / \frac{R_{T L_{6 \circ} \mathrm{Co}}}{\text { dose }}(1)
$$

$\frac{R_{T L_{E 0} C_{0}}}{\text { dose }}$ corresponde ao valor obtido quando empregado a irradiação de referência com o 60Co.

Tabela 3a: Resposta TL e fator de correção obtidos para placas de PMMA.

\begin{tabular}{|c|c|c|c|}
\hline & $\begin{array}{l}\text { Qualidades } \\
\text { de Radiação }\end{array}$ & Resposta TL $(\mu \mathrm{C}) \pm 0,2 \%$ & $\begin{array}{l}\text { Fator de dependên- } \\
\text { cia energética }\end{array}$ \\
\hline & ${ }^{60} \mathrm{Co}$ & $1,76 \mathrm{E}-05 \pm 3,52 \mathrm{E}-08$ & 1 \\
\hline & ${ }^{137} \mathrm{Cs}$ & $1,99 \mathrm{E}-05 \pm 3,98 \mathrm{E}-08$ & 1,13 \\
\hline & N150 & $1,22 \mathrm{E}-05 \pm 2,44 \mathrm{E}-08$ & 2,09 \\
\hline & RQR10 & $1,29 \mathrm{E}-04 \pm 2,58 \mathrm{E}-08$ & 7,36 \\
\hline & $\begin{array}{l}\text { QuARARAes } \\
\text { de RBQRọão }\end{array}$ & 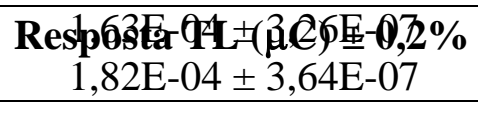 & $\begin{array}{c}\text { Fat } \partial \text { rQde dependên- } \\
\text { ci®, Anergética }\end{array}$ \\
\hline & ${ }^{60} \mathrm{Co}$ & $1,80 \mathrm{E}-05 \pm 3,6 \mathrm{E}-08$ & 1 \\
\hline Ta- & ${ }^{137} \mathrm{Cs}$ & $2,03 \mathrm{E}-05 \pm 4,06 \mathrm{E}-08$ & 1,13 \\
\hline $\begin{array}{c}\text { 3b: } \\
\text { posta }\end{array}$ & N150 & $1,26 \mathrm{E}-05 \pm 2,52 \mathrm{E}-08$ & 2,1 \\
\hline fator & & & \\
\hline cor- & & & \\
\hline obti- & & & \\
\hline
\end{tabular}

para placas de PLA. 


\begin{tabular}{l|c|c} 
RQR10 & $1,37 \mathrm{E}-04 \pm 2,74 \mathrm{E}-07$ & 7,6 \\
RQR5 & $1,73 \mathrm{E}-04 \pm 3,46 \mathrm{E}-07$ & 9,64 \\
RQR3 & $1,70 \mathrm{E}-04 \pm 3,40 \mathrm{E}-07$ & 9,62 \\
\hline
\end{tabular}

A figura 6 apresenta as curvas de dependência energética da resposta TL do CaSO4:Dy empregando os dois tipos de placas.

Figura 6. Curvas de dependência energética da resposta TL do CaSO4:Dy empregando os dois tipos de placas.

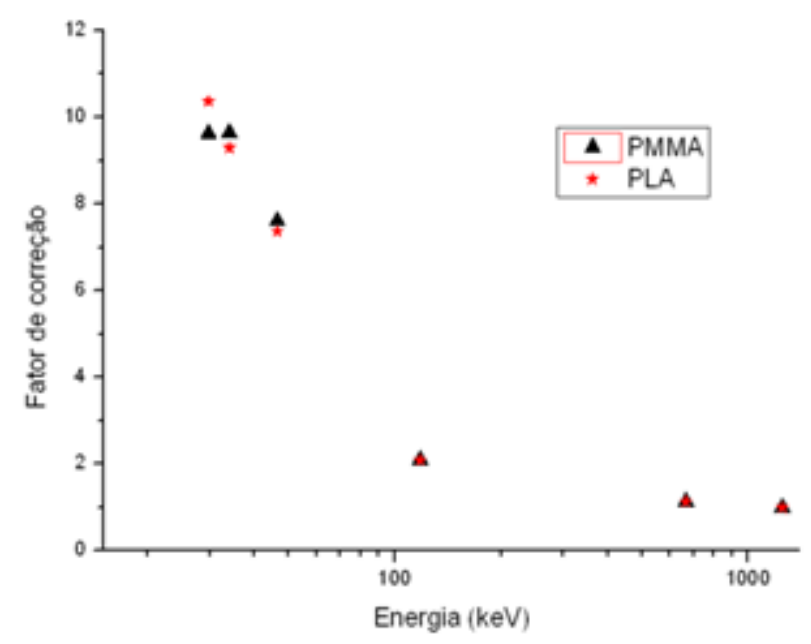

A partir da curva de calibração (figura 5) foi calculado o fator de calibração do lote de dosímetros, o qual foi empregado na conversão da resposta TL $(\mu \mathrm{C})$ para a dose $(\mathrm{Gy})$. Os valores de dose obtidos para os dosímetros irradiados (empregando as placas de PMMA e PLA) são mostrados na tabela 4 , juntamente com suas respectivas incertezas obtidas por meio da propagação 
de incertezas, onde foram consideradas as incertezas no fator de calibração da fonte de 60Co e no valor das doses de raios $\mathrm{X}$, fornecidas pelo Laboratório, bem como do fator de calibração do lote.

Os valores das tabela 4 foram com a dose node radiação gama radiativa de refesido calculada dade de radiação) centual entre os \begin{tabular}{l|c|c|c}
\hline \multicolumn{4}{c}{ Dose (mGy) } \\
\hline $\begin{array}{l}\text { Qualidades de } \\
\text { Radiação }\end{array}$ & PMMA & PLA & $\begin{array}{c}\text { Diferença } \\
\text { percentual }\end{array}$ \\
\hline${ }^{60}$ Co & $28,3 \pm 0,04 \%$ & $25,5 \pm 0,04 \%$ & 9,89 \\
${ }^{137}$ Cs & $28,3 \pm 0,05 \%$ & $25,5 \pm 0,03 \%$ & 9,89 \\
N150 & $9,4 \pm 0,03 \%$ & $8,48 \pm 0,05 \%$ & 9,79 \\
RQR10 & $28,3 \pm 0,21 \%$ & $25,5 \pm 0,42 \%$ & 9,89
\end{tabular} doses contidos na normalizados PMMA e o PLA, equação 2. Sendo minal de 30 mGy do ${ }^{60} \mathrm{Co}$ (fonte rência), tendo (para cada qualia diferença pervalores para o por meio da as diferenças menores que $10 \%$.

$$
D i f_{\%}=\left[\frac{D_{P M M A}-D_{P L A}}{D_{P M M A}}\right] * 100
$$

Tabela 4: Doses calculadas empregando placas de PMMA e PLA, relativas a uma dose nominal de radiação gama de $30 \mathrm{mGy}$. 


\begin{tabular}{l|l|l|l} 
RQR5 & $28,3 \pm 0,77 \%$ & $25,5 \pm 1,42 \%$ & 9,89 \\
RQR3 & $28,3 \pm 0,52 \%$ & $25,5 \pm 0,46 \%$ & 9,89 \\
\hline
\end{tabular}

Conforme o manual do fabricante, a malha impressa não é totalmente sólida, de modo a existir es-paços não preenchidos por PLA (figura 4). Como pode ser observado na tabela 4, as doses avaliadas para as placas de PLA são inferiores àquelas obtidas para o PMMA. Nas tabelas 3a e $3 \mathrm{~b}$ os fatores de correção para a dependência energética nas qualidades de raios $\mathrm{X}$ diagnóstico (exceto RQR3) mostram que o PMMA, com a espessura adequada para o equilíbrio eletrônico para radiação do ${ }^{60} \mathrm{Co}$, apresenta fatores de dependência energética sempre maiores que para o PLA, sendo um demonstrativo de que a espessura de $3 \mathrm{~mm}$ de PLA não é suficiente para fornecer condição de equilíbrio eletrônico para radiação do ${ }^{60} \mathrm{Co}$, com consequente subestimativa da dose. Para a qualidade RQR 3, há o indicativo de que com a baixa energia efetiva do feixe (29,7keV), e considerando a qualidade da im-pressão utilizada, a placa de PLA impressa irá apresentar uma atenuação diferenciada, provocando dessa forma uma maior dependência energética da resposta TL do CaSO4:Dy. Desse modo, é necessário o uso cauteloso e uma melhor avaliação das espessuras das placas de PLA impressas, de modo a que seja corrigida a influência da qualidade da impressão na equivalência do PLA ao polimetilmetacrilato.

\section{AGRADECIMENTO}

Os autores agradecem à FAPESP e ao CNPq pelo apoio financeiro, à empresa Eletrocell CIETEC/IPEN pela manutenção da impressora 3D e ao LCI/IPEN pelas irradiações realizadas. 


\section{REFERÊNCIAS}

1. Journal

CAMPOS, L.L.; LIMA, M.F. Dosimetric Properties of CaSO4 : Dy + Teflon Pellets Produced at IPEN.- Rad. Prot. Dosim. 14, 4 (1986 ) 333.

Nunes, M.G., Campos, L. L.Study of CaSO4:Dy and LiF:Mg,Ti Detectors TL Response to Electron Radiation Using a SW Solid Water Phantom. Rad. Measurement. 43 (2008) 459 - 462

\section{Book}

CAMERON, J.R; SUNTHARALINGAM, N.; KENNEY, G.N. Thermoluminescent Dosimetry. Madison, Wisconsin: The University of Wisconsin Press, Ltd., 1968.

TAYLOR, J.R. Introdução à Análise de Erros: O Estudo de Incertezas em Medições. Porto Alegre, Rio Grande do Sul: Bookman Companhia Editora Ltda., 2012.

CUNICO, M. W. M. Desenvolvimento de nova tecnologia de manufatura aditiva baseado em formação seletiva de compósito. 2013. Tese (Doutorado) - Universidade de São Paulo, São Carlos, 2013.

\section{Report}

ICRU - International Commission on Radiation Units and Measurements. Fundamental quantities and units for ionizing radiation. ICRU Report 60, Bethesda: ICRU, 1998. 24p.

\section{Web site}


UP! 3D Brasil. UP! User Manual. Disponível em:< http://www.bcstecnologia.com.br/cariboost_files/UP_Plus_2_User_Manual-PT_BRV2.pdf>. Last accessed:01 nov 2015

\section{Conferences Proceedings}

VENEZIANI, G. R.; CORREA, E.L.; POTIENS, M.P.A.; CAMPOS, L.L.. Attenuation coefficient determination of printed abs and pla samples in diagnostic radiology standard beams. In: $8^{\circ}$ Congresso Brasileiro de Metrologia. 29 novembro à 4 de dezembro, 2015. Bento Gonçalves, RS. 